\title{
Dark Matter searches with the ATLAS Detector
}

\author{
Charles William Kalderon* ${ }^{* \dagger}$ \\ Lund University, Sweden \\ E-mail: william.kalderon@cern.ch
}

\begin{abstract}
As evinced by multiple astrophysical measurements, a large fraction of the matter in the Universe is in the form of a dark, non-baryonic component. If dark matter interacts weakly with Standard Model particles it could be produced at the LHC, escaping the ATLAS detector and thus leaving a signature of large missing transverse momentum. If this interaction is mediated by a kinematically accessible mediator, then that mediator can also give rise to a dijet resonance signature. The latest results of these dijet resonance searches are presented, and their limitations and future prospects discussed.
\end{abstract}

XXVI International Workshop on Deep-Inelastic Scattering and Related Subjects (DIS2018) 16-20 April 2018

Kobe, Japan

\footnotetext{
* Speaker.

${ }^{\dagger}$ On behalf of the ATLAS Collaboration.
} 


\section{Introduction}

There is strong astronomical and cosmological evidence for the near-ubiquitous presence of dark matter in the wider universe, on a variety of scales. A compelling hypothesis is that of "WIMP" dark matter - where the dark matter present in the universe takes the form of a weaklyinteracting massive particle, i.e. with a mass around the $\mathrm{GeV}$ scale and couplings to standard model particles of weak-scale strength [1]. There is however as-yet no conclusive evidence of the existence of such dark matter through either indirect or direct detection.

\section{LHC search methods}

If dark matter (DM) couples to standard model (SM) particles, in particular to quarks, then it can be produced at the LHC. In the case of simple pair-production of dark matter, it would leave no discernible signature at all in the ATLAS detector [2], so the challenge becomes one of searching for the invisible, which can be resolved in two ways.

The first option is to require additional activity in the event through for instance initial state radiation (ISR) of a quark, photon, vector boson, Higgs, etc. This initial state radiation is itself visible to ATLAS and allows an event to be triggered on and selected over backgrounds, and additionally provides a recoil momentum to the dark matter particles which manifests as missing transverse momentum. Analyses searching for this type of signature - a single particle balancing missing transverse momentum - are known as 'mono- $X$ ' searches, and there have been many ATLAS results of this kind over the past year: jet [3], photon [4], $Z \rightarrow \ell \ell$ [5], $W / Z \rightarrow q q$ [6], $h \rightarrow b b$ [7], $h \rightarrow \gamma \gamma$ [8]. Additionally, particularly in the case of scalar mediators between dark matter and the standard model, third-generation couplings are enhanced and dark matter can be produced in association with one or two top or bottom quarks [9].

The second option is to probe the interaction between dark matter and SM particles. As an example of such a strategy, the most relevant interactions at LHC energy scales can be represented by a simplified model of dark matter production with a mediator particle $Z^{\prime}$ between quarks and dark matter. One can then search for this $Z^{\prime}$ directly, through its decays back to quarks that yield resonant signatures in the dijet invariant mass ${ }^{1}$. Such a $Z^{\prime}$ particle can also, in more complicated models, be produced along with a dark matter pair: such a scenario is covered in ref. [10].

The simplified dark matter model mentioned above has two key parameters: the mass of the mediator $m_{Z^{\prime}}$ and its coupling to quarks $g_{q}$. In simple dijet resonance interpretations the dark matter mass $m_{\mathrm{DM}}$ is set very high such that it is kinematically inaccessible, in order to factorise the visible and invisible decays of the $Z^{\prime}$, and its coupling to the $Z^{\prime} g_{\mathrm{DM}}$ is therefore of limited relevance.

\section{ATLAS resonance search limits and constraints}

The limits from dijet resonance searches to such $Z^{\prime}$ models from dijet resonance searches in 2013 is shown in fig. 1 . Here $Z_{B}$ is identified as the mediator $Z^{\prime}$ and $g_{B}$ is six times the coupling of the mediator to quarks $g_{q}$. The red line is the exclusion limit from an ATLAS dijet resonance search with $20.3 \mathrm{fb}^{-1}$ of $8 \mathrm{TeV}$ data collected in 2012 [11].

\footnotetext{
${ }^{1}$ The $Z^{\prime}$ could also couple to leptons, causing dilepton resonances, which in general are advantageous to search for at hadron colliders due to the lower backgrounds. This talk however focuses on searches for hadronic resonances.
} 
The equivalent run 2 ATLAS search [13], with $37.0 \mathrm{fb}^{-1}$ of $13 \mathrm{TeV}$ data collected in 2015 and 2016, excludes values $g_{q}$ below 0.07 for $m_{Z^{\prime}}=1.5 \mathrm{TeV}$, compared to around 0.15 for the run 1 result. However it does not set limits below $m_{Z^{\prime}}=1.5 \mathrm{TeV}$, due to trigger constraints, which are also why the run 1 result starts becoming weaker below $1 \mathrm{TeV}$. Below this mass the search used prescaled triggers (only a fraction of events accepted by a trigger are recorded) and a delayed stream (events accepted by some triggers were written to a separate stream that was not reconstructed until computing re-

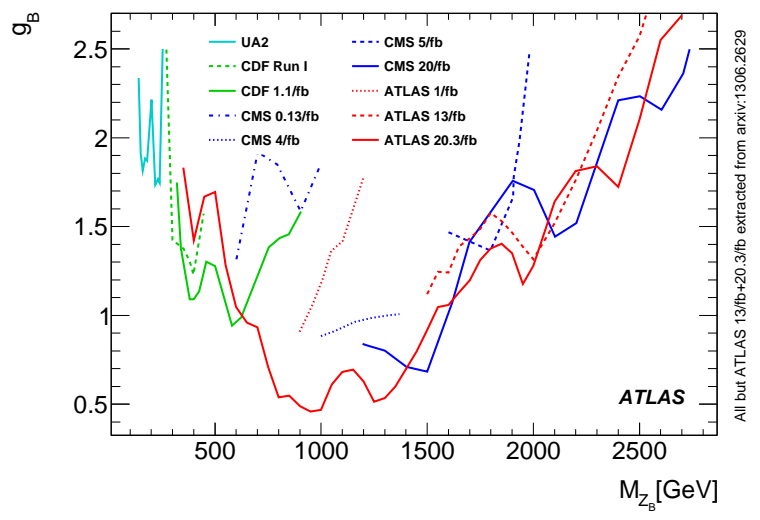

Figure 1: Summary of $95 \%$ C.L. limits on coupling $g_{B}$ vs $m_{Z_{B}}$ from the UA2, CDF, CMS and ATLAS experiments as of the end of run $1[11,12]$. sources became available over the 2013-2014 shutdown).

The ATLAS trigger system [14] consists of two parts: a first level (L1) to reduce the event rate from the $30 \mathrm{MHz}$ delivered by the $\mathrm{LHC}^{2}$ to the $\sim 100 \mathrm{kHz}$ that the subdetectors can be read out at, and a 'high level trigger' (HLT) that reduces this to $\sim 1-1.5 \mathrm{kHz}$ for offline storage, limited by the total storage and processing cost. Of this, $\sim 20-40 \mathrm{~Hz}$ is dedicated to the single jet trigger used by this dijet resonance search [15]. Since this rate constraint is fixed, the jet trigger $p_{\mathrm{T}}$ threshold must be raised as instantaneous luminosity increases (it is observed that the trigger rate is proportional to $p_{\mathrm{T}}^{-5}$ ). In 2016, the trigger selected events containing a jet with $E_{\mathrm{T}}>380 \mathrm{GeV}$, which was fully efficient in selecting jets with $p_{\mathrm{T}}>440 \mathrm{GeV}$ offline, and it is this which sets the lower bound on $Z^{\prime}$ mass sensitivity.

\section{Overcoming trigger limitations: Initial State Radiation}

These trigger limitations can be overcome by requiring the presence of initial state radiation, either a photon or a jet, which if sufficiently high transverse momentum ( $380 \mathrm{GeV}$ for a jet, $140 \mathrm{GeV}$ for a photon) can be triggered on. This removes the $p_{\mathrm{T}}$ constraints on the resonance jets, which can therefore be much softer. This strategy is used in ref. [16], allowing $Z^{\prime}$ masses down to $200 \mathrm{GeV}$ to be probed.

Below this mass, the resonance jets tend to merge into a single jet and the above search, for a dijet resonance, loses sensitivity. This lower mass region is tackled by a search for localised excesses in the single jet mass spectrum of large-radius jets, which capture both quarks from the resonance decay [17]. This uses the jet substructure variable $\tau_{21}$ to distinguish 2-subjet signal from single-subjet QCD background, modified using the "designed decorrelated tagger" method to decorrelate it from jet mass in order that the $\tau_{21}$ selection does not overly bias the jet mass distribution. The dominant QCD background is estimated with a data-driven method based on inverting this $\tau_{21}^{D D T}$ selection, validated on the $W / Z$ peak. The search is then performed in wide mass windows, each containing the bulk of the resonance (fig. 2), rather than searching for excesses of unknown width as in other dijet searches.

\footnotetext{
${ }^{2}$ a $25 \mathrm{~ns}$ bunch spacing gives $40 \mathrm{MHz}$, but the ring is not full
} 
The exclusion limits of this search (turquoise) and the 'resolved' dijet + ISR search (red and purple) are shown in fig. 3. The 'merged' search provides sensitivity from 100 to $200 \mathrm{GeV}$, while the 'resolved' one does so from $200 \mathrm{GeV}$ to $1 \mathrm{TeV}$ (with extra sensitivity in the jet ISR channel from 350 to $450 \mathrm{GeV}$ ). However, these have much lower $g_{q}$ sensitivity than the dijet resonance search (dark blue) -0.15 vs 0.07 - due to requiring an energetic radiated jet or photon. There is also a gap between these searches' sensitivities between 1 and $1.5 \mathrm{TeV}^{3}$. These limitations are overcome by a technique new to ATLAS - triggerlevel analysis.

\section{Overcoming}

\section{trigger limitations: Trigger-Level Analysis}

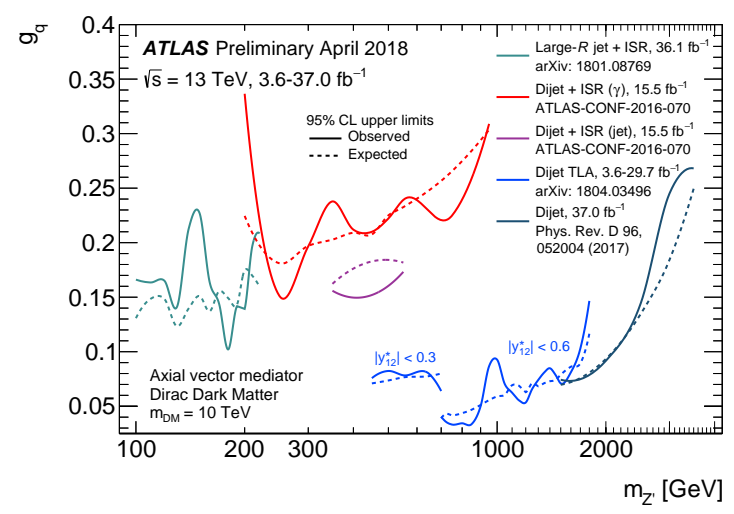

Figure 3: Summary plot of ATLAS bounds in the $m_{Z^{\prime}}-g_{q}$ plane from dijet searches in run 2 data [19].

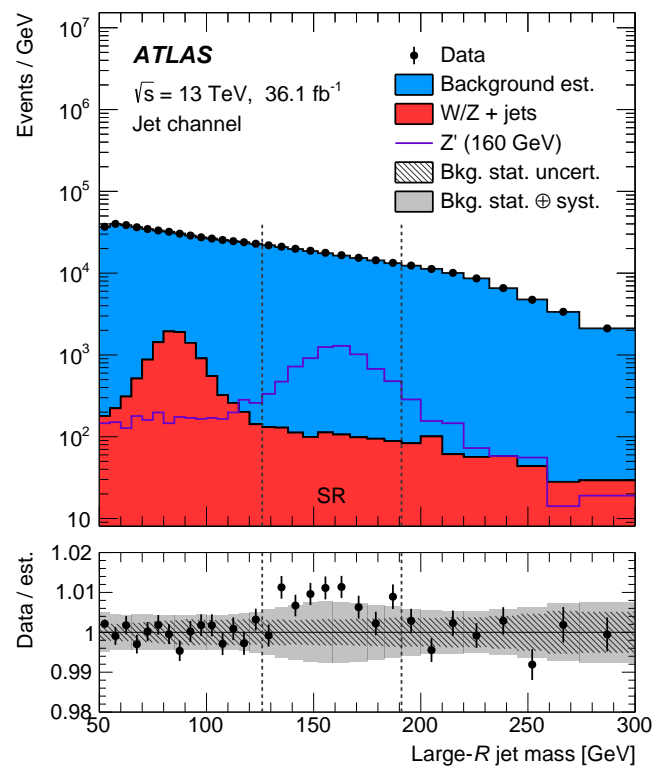

Figure 2: Distribution of large- $R$ jet mass, including a signal with $m_{Z^{\prime}}=160 \mathrm{GeV}$ and $g_{q}=$ 0.5 [17].

The main driver of the $1.5 \mathrm{kHz}$ limit on the HLT output rate is the offline storage and processing cost. Hence it is more strictly a bandwidth limit rather than a pure rate limit. Since a dijet resonance search only requires jets (and not e.g. leptons), and since jets are well reconstructed in the trigger, a new data stream can be provided that contains only trigger jets $(0.5 \%$ of normal event size $)$ at a high rate $(1-3 \mathrm{kHz})$ with minimal bandwidth impact. This idea was pioneered at the LHC by CMS [20, 21] and LHCb [22], and was first implemented for ATLAS in 2015 under the name 'trigger-level analysis' (TLA) [23].

This new data stream allowed all events with leading jet $p_{\mathrm{T}}$ above $220 \mathrm{GeV}$ to be included in the search, meaning that the mass spectrum analyses started at $531 \mathrm{GeV}$ rather than $1100 \mathrm{GeV}$ for the offline search (fig. 4).

In addition to trigger jet four-momenta, the trigger-level analysis stream contains sufficient information to be able to redo jet calibration. The procedure followed is as close as possible to that for offline jets [24], with some parts re-derived since TLA data lacks track information, as shown in fig. 5. The result of this specific calibration is excellent agreement between offline and recalibrated trigger dijet mass $m_{j j}$ (the mean difference is less than $0.5 \%$ for all $m_{j j}$ considered).

The search is performed through fitting a smooth functional form to the data. However, the unprecedented amount of data in the $m_{j j}$ spectrum meant that the known functions could not

\footnotetext{
${ }^{3}$ In run 1 this area is covered by dijet searches (see fig. 1 ) and in run 2 to a lesser degree by di- $b$-jet resonances [18].
} 


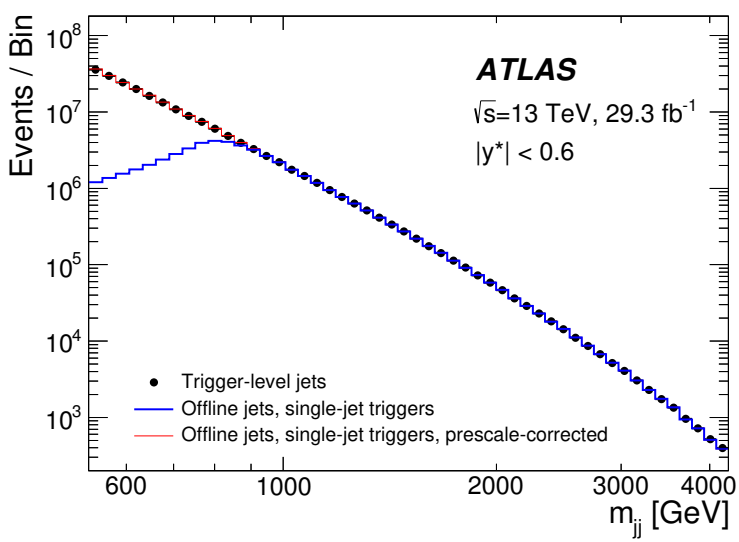

Figure 4: Comparison between the number of dijet events in the data used by the TLA (black points), the number of events selected by any single-jet trigger (thicker, blue line), and the events selected by singlejet triggers but corrected for the trigger prescale factors (thinner, red line) as a function of $m_{j j}$ [23]. adequately describe the data over the whole mass range. Thus the background estimation at each mass was obtained by fitting a subrange of the spectrum around that mass (see animation at [25]).

Following this procedure, no significant excesses were observed, and the limits shown in blue in fig. 3 above were obtained. The TLA greatly improves sensitivity over dijet+ISR, despite the lower mass TLA signal region (using data collected with a lower threshold trigger at the beginning of the 2016 data-taking period, and a tighter $|y *|=\frac{1}{2}\left|y_{1}-y_{2}\right|$ selection to lower the $Z^{\prime}$ mass cutoff) using far less integrated luminosity. The higher mass TLA signal region fills in the gap in ATLAS run 2 sensitivity to these $Z^{\prime}$ models and ensures ATLAS run 2 limits now surpass pre-LHC ones (fig. 1) everywhere.

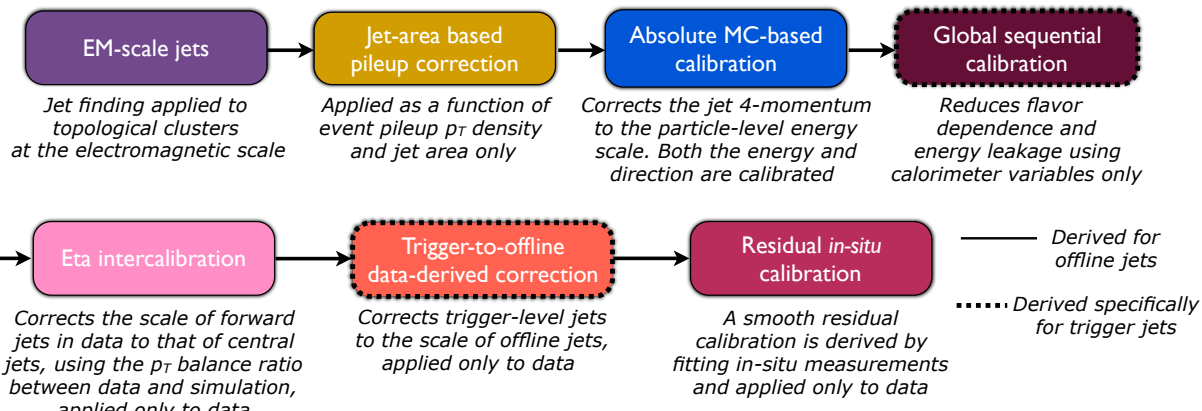

Figure 5: Calibration stages for trigger-level jets, each applied to the four-momentum of the jet [23].

\section{Future prospects}

The $p p \rightarrow Z^{\prime}$ cross-section is proportional to $g_{q}^{2}$, and in a statistically limited search the cross-

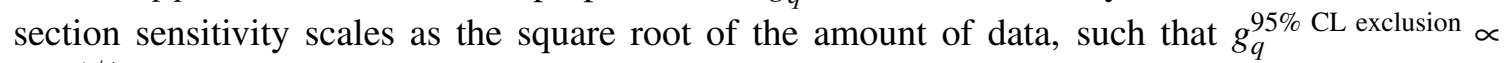
data $\left.^{1 / 4}\right)$. With this scaling a total run 2 dataset of $120 \mathrm{fb}^{-1}$ gives $g_{q}$ limit improvements of a factor of 1.3 - 1.7, but each of the searches mentioned here can improve significantly beyond that.

For the trigger-level analysis, a major improvement in the remainder of run 2 is to take advantage of unused L1 rate towards end of LHC fills to run lower threshold triggers $-50 \mathrm{GeV}$ at L1 vs 75 and 100 in 2016 - allowing lower masses to be probed. In run 3 (i.e. from 2021), new hardware will allow improved reconstruction of L1 objects meaning that the TLA will be able to access lower masses for a given recording rate. Additionally, the fast tracker (FTK) [26, 27] will provide full tracking at the HLT, allowing pileup rejection and improved jet calibration [28]. 
For the resolved dijet + ISR search, the higher trigger thresholds required by the increased instantaneous luminosity in 2017 and 2018 are mitigated by improved jet trigger performance such that in 2017 the offline jet selection can remain the same as in 2016. Work on combinatorics in jet channel - i.e. which of the three jets are from the resonance and which is ISR - can improve mass reach (and sensitivity for some masses); and there is potential for using the TLA technique in run 3 with the FTK to suppress pileup at low jet $p_{\mathrm{T}}$, which would improve sensitivity thanks to imposing weaker ISR constraints and hence reducing the loss in signal acceptance.

For the merged dijet + ISR search, new trigger strategies for large radius jets, including substructure information in the trigger (some 2017 triggers select on mass), will lead to greater signal acceptance. Optimised grooming methods as described in ref. [29] promise improved signal vs background discrimination, as do improvements in jet substructure resolution thanks to track information being included in jet reconstruction inputs [30].

\section{Complementarity}

As can be seen in fig. 6, mono$\mathrm{X}$ and resonance searches complement each other to cover a wide range of the $m_{\mathrm{DM}}-m_{Z^{\prime}}$ plane, with mono-X searches being more sensitive for low masses. There is also complementarity between both sets of collider searches and direct detection. In both cases the interplay between different searches strongly depends on the model considered, see ref. [19] for other models and continually updated versions of these plots.

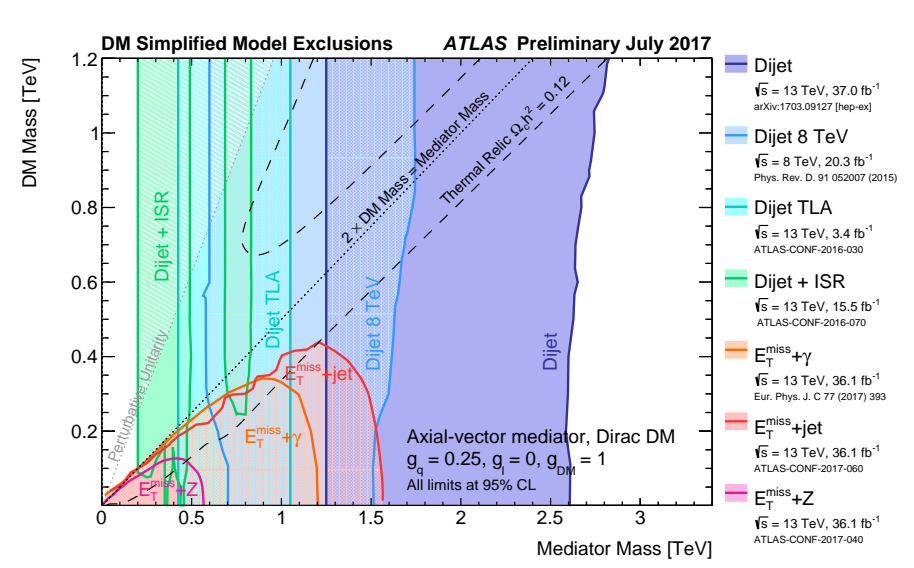

Figure 6: Regions in a dark matter mass-mediator mass plane excluded at $95 \%$ CL by a selection of ATLAS dark matter searches, for one possible interaction between the Standard Model and dark matter, the leptophobic axial-vector mediator as described in ref. [31]. From [19].

\section{Conclusions}

There are a broad set of approaches to searching for Dark Matter with ATLAS, including a variety of new techniques targeting low mass mediators which lead to dijet resonances. These make use of initial state radiation to evade trigger limitations, and substructure to take this approach into the very low mass regime where the resonance jets merge. ATLAS has for the first time made use of a method pioneered by LHCb and CMS to make the best use of the trigger system and perform a dijet analysis with partial events, significantly extending sensitivity to these models. This technique may also help with significant computing and storage pressures in the future as LHC performance improves.

These new techniques can all take advantage of upcoming trigger upgrades, which will result in run 3 searches with sensitivity improved much more than would be expected from integrated luminosity scaling alone. 


\section{References}

[1] G. Bertone, D. Hooper and J. Silk, Particle dark matter: Evidence, candidates and constraints, Phys. Rept. 405 (2005) 279 [hep-ph / 0404175$].$

[2] ATLAS Collaboration, The ATLAS Experiment at the CERN Large Hadron Collider, JINST 3 (2008) S08003.

[3] ATLAS Collaboration, Search for dark matter and other new phenomena in events with an energetic jet and large missing transverse momentum using the ATLAS detector, JHEP 01 (2018) 126 [1711.03301].

[4] ATLAS Collaboration, Search for dark matter at $\sqrt{s}=13 \mathrm{TeV}$ in final states containing an energetic photon and large missing transverse momentum with the ATLAS detector, Eur. Phys. J. C 77 (2017) 393 [1704.03848].

[5] ATLAS Collaboration, Search for an invisibly decaying Higgs boson or dark matter candidates produced in association with a $Z$ boson in pp collisions at $\sqrt{s}=13 \mathrm{TeV}$ with the ATLAS detector, Phys. Lett. B 776 (2018) 318 [1708.09624].

[6] ATLAS Collaboration, Search for dark matter produced in association with a hadronically decaying vector boson in pp collisions at $\sqrt{s}=13 \mathrm{TeV}$ with the ATLAS detector, Phys. Lett. B 763 (2016) 251 [1608.02372].

[7] ATLAS Collaboration, Search for Dark Matter Produced in Association with a Higgs Boson Decaying to $b \bar{b}$ using $36 \mathrm{fb}^{-1}$ of pp collisions at $\sqrt{s}=13 \mathrm{TeV}$ with the ATLAS Detector, Phys. Rev. Lett. 119 (2017) 181804 [1707.01302].

[8] ATLAS Collaboration, Search for dark matter in association with a Higgs boson decaying to two photons at $\sqrt{s}=13 \mathrm{TeV}$ with the ATLAS detector, Phys. Rev. D 96 (2017) 112004 [1706. 03948 ].

[9] ATLAS Collaboration, Search for top-squark pair production in final states with one lepton, jets, and missing transverse momentum using $36 \mathrm{fb}^{-1}$ of $\sqrt{s}=13 \mathrm{TeV}$ pp collision data with the ATLAS detector, 1711.11520 .

[10] ATLAS Collaboration, Search for dark matter in events with a hadronically decaying vector boson and missing transverse momentum in pp collisions at $\sqrt{s}=13 \mathrm{TeV}$ with the ATLAS detector, ATLAS-CONF-2018-005.

[11] ATLAS Collaboration, Search for new phenomena in the dijet mass distribution using pp collision data at $\sqrt{s}=8 \mathrm{TeV}$ with the ATLAS detector, Phys. Rev. D 91 (2015) 052007 [1407.1376].

[12] B. A. Dobrescu and F. Yu, Coupling-mass mapping of dijet peak searches, Phys. Rev. D88 (2013) 035021 [1306.2629].

[13] ATLAS Collaboration, Search for new phenomena in dijet events using $37 \mathrm{fb}^{-1}$ of pp collision data collected at $\sqrt{s}=13 \mathrm{TeV}$ with the ATLAS detector, Phys. Rev. D 96 (2017) 052004 [1703.09127].

[14] ATLAS Collaboration, Performance of the ATLAS Trigger System in 2015, Eur. Phys. J. C 77 (2017) 317 [1611.09661].

[15] ATLAS Collaboration, Trigger Menu in 2016, ATL-DAQ-PUB-2017-001.

[16] ATLAS Collaboration, Search for new light resonances decaying to jet pairs and produced in association with a photon or a jet in proton-proton collisions at $\sqrt{s}=13$ TeV with the ATLAS detector, ATLAS-CONF-2016-070. 
[17] ATLAS Collaboration, Search for light resonances decaying to boosted quark pairs and produced in association with a photon or a jet in proton-proton collisions at $\sqrt{s}=13$ TeV with the ATLAS detector, 1801.08769 .

[18] ATLAS Collaboration, Search for resonances in the mass distribution of jet pairs with one or two jets identified as b-jets in proton-proton collisions at $\sqrt{s}=13 \mathrm{TeV}$ with the ATLAS detector, 1805.09299.

[19] ATLAS Collaboration, Summary plots from the atlas exotic physics group, https://atlas.web.cern.ch/Atlas/GROUPS/PHYSICS/CombinedSummaryPlots/EXOTICS/index.html.

[20] CMS Collaboration, Search for Narrow Resonances in Dijet Final States at $\sqrt{s}=8$ TeV with the Novel CMS Technique of Data Scouting, Phys. Rev. Lett. 117 (2016) 031802 [1604.08907].

[21] CMS Collaboration, Search for dijet resonances in proton-proton collisions at $\sqrt{s}=13$ TeV and constraints on dark matter and other models, Phys. Lett. B 769 (2017) 520 [1611.03568].

[22] LHCb Collaboration, Tesla : an application for real-time data analysis in High Energy Physics, Comput. Phys. Commun. 208 (2016) 35 [1604.05596].

[23] ATLAS Collaboration, Search for low-mass dijet resonances using trigger-level jets with the ATLAS detector in pp collisions at $\sqrt{s}=13 \mathrm{TeV}, 1804.03496$.

[24] ATLAS Collaboration, Monte Carlo Calibration and Combination of In-situ Measurements of Jet Energy Scale, Jet Energy Resolution and Jet Mass in ATLAS, ATLAS-CONF-2015-037.

[25] ATLAS Collaboration, Animation of the fit procedure used for the trigger-level analysis, https://atlas.web.cern.ch/Atlas/GROUPS/PHYSICS/PAPERS/EXOT-2016-20/figaux_06.png.

[26] ATLAS Collaboration, Technical Design Report for the Phase-I Upgrade of the ATLAS TDAQ System, CERN-LHCC-2013-018, ATLAS-TDR-023.

[27] J. Anderson, A. Andreani, A. Andreazza, A. Annovi, M. Atkinson, B. Auerbach et al., FTK: a Fast Track Trigger for ATLAS, JINST 7 (2012) C10002.

[28] ATLAS Collaboration, Trigger-object Level Analysis with the ATLAS detector at the Large Hadron Collider: summary and perspectives, ATL-DAQ-PUB-2017-003.

[29] ATLAS Collaboration, Impact of Alternative Inputs and Grooming Methods on Large-R Jet Reconstruction in ATLAS, ATL-PHYS-PUB-2017-020.

[30] ATLAS Collaboration, Improving jet substructure performance in ATLAS using Track-CaloClusters, ATL-PHYS-PUB-2017-015.

[31] A. Albert et al., Recommendations of the LHC Dark Matter Working Group: Comparing LHC searches for heavy mediators of dark matter production in visible and invisible decay channels, 1703.05703 . 